\title{
La red de transporte masivo como soporte del sistema de espacios abiertos metropolitanos. Caso de estudio: La Línea 2 del Metro de Lima.
}

\author{
Susana López Varela ${ }^{1}$, Joan Moreno Sanz ${ }^{2}$ \\ ${ }^{1}$ Departamento de Urbanismo, Pontificia Universidad Católica del Perú, PUCP. \\ ${ }^{2}$ Departamento de Urbanismo y Ordenación del Territorio, Universidad Politécnica de Catalunya. \\ E-mail: 1'slopezv@pucp.edu.pe, ${ }^{2}$ joan.moreno@upc.edu
}

\begin{abstract}
Resumen. La gestión de la movilidad es clave en la prosperidad social y económica de las ciudades. El desarrollo urbano disperso y monofuncional ha favorecido la dependencia de sistemas de locomoción individuales. Las consecuencias son: exclusión social, segregación espacial, ocupación del espacio público, degradación ambiental, etc. La planificación urbana orientada a la movilidad sostenible podría contribuir a la mejora de la eficiencia de la red de transporte y vertebrar un ecosistema de espacios abiertos accesible e inclusivo.

El objetivo principal de esta comunicación es evaluar el papel de la red de transporte masivo como elemento vertebrador de un sistema de espacios abiertos conectados e inclusivos. El caso de estudio escogido es el proyecto de la línea 2 del Metro de Lima. Metodológicamente, se ha considerado la relación entre la morfología urbana, la composición social, la mixtura de actividad y la calidad de los espacios libres, en el entorno de la línea. La planificación integrada de la infraestructura verde y gris podría contribuir a crear un sistema metropolitano de espacios abiertos accesibles e inclusivos.
\end{abstract}

Palabras clave: Movilidad urbana, espacios abiertos, desarrollo urbano sostenible, transporte masivo, Lima

\section{Introducción}

En América Latina, la población urbana supone el $81 \%$ del total (Noticias ONU, 2018), por lo tanto, las ciudades son piezas fundamentales en la construcción de un futuro social, ambiental y económicamente sostenible. Desde el punto de vista de la movilidad, la Nueva Agenda Urbana - Hábitat III apuesta por un desarrollo orientado al transporte, incorporando los planes de movilidad en la planificación urbana y territorial, y promoviendo el desarrollo de un sistema integrado de transporte público, accesible, eficiente y asequible frente al transporte privado. El objetivo es reducir la congestión y los desplazamientos al tiempo que mejorar la eficiencia, la conectividad, la accesibilidad y la salud; en definitiva, la calidad de vida de los ciudadanos.

En lo concerniente a los espacios abiertos, la Nueva Agenda Urbana se compromete a promover la creación de redes de espacios públicos de calidad, conectados y distribuidos, seguros, inclusivos, accesibles, verdes y destinados a fines múltiples, permitiendo así incrementar la resiliencia urbana frente al cambio climático, mejorar la salud físicamental y calidad del aire, reducir el ruido, y promover ciudades más atractivas y habitables.

\section{Los espacios abiertos como infraestructura.}

A pesar de los enormes beneficios que los espacios abiertos generan para la urbe y sus ciudadanos -funcionalidad ambiental, satisfacción de las necesidades de ocio y 
provisión de servicios ecológicos- (Tratalos et al., 2007), su papel en el sistema urbano ha tenido con frecuencia un rol pasivo. El que hayan sido entendidos como fragmentos aislados y desconectados entre sí, muchas veces inaccesibles y ocupando lugares residuales, ha propiciado este entendimiento (Feria \& Santiago, 2009).

Sin embargo, el hecho demostrado de que variables como la distribución, conectividad y accesibilidad son determinantes para la calidad de los servicios que los espacios abiertos prestan a la ciudad (Barbosa, 2007; Figueroa, 2009), ha conducido a un replanteamiento en la manera como concebir estos y su planificación: ser entendidos desde una concepción sistémica o de red y por tanto, ser reconocidos como un subsistema fundamental en la vertebración territorial de ámbitos urbanos y metropolitanos (Cruz, 2017).

Tratados metodológicos como el modelo Matriz-Parche-Corredor de Forman \& Gordon (1986) entendieron la conectividad como herramienta clave para entender el funcionamiento de la ciudad; pero será a partir de la introducción del concepto de Infraestructura Verde cuando se dé un paso más en su conceptualización. Esta red de espacios abiertos, diseñada y gestionada como un recurso multifuncional, capaz de suministrar a la comunidad la calidad de vida y los servicios ecosistémicos necesarios acordes con criterios de sostenibilidad (Natural England, 2009; EEA, 2011), reconocerá el rango infraestructural de los espacios abiertos, equiparándolos a los sistemas que constituyen el principal armazón urbano: transportes, equipamientos y servicios. Asimismo, y para que su carácter multifuncional como prestadora de servicios ecosistémicos (MEA, 2005; Natural England, 2009) se haga efectivo, resultará esencial incluir las "infraestructuras grises" (redes, servicios públicos y corredores de transporte) dentro de su engranaje (Thomas \& Littlewood, 2010).

La conjunción de dos conceptos antagónicos ("infraestructura" y "verde"), además de permitir atender simultáneamente las múltiples necesidades que tiene la ciudad, mejorando así sus condiciones físicas, sociales y ambientales (Pons: 162, 2016), ayudaría a reconciliar el desarrollo urbano, asegurando una gestión sostenible de las áreas metropolitanas a medio y largo plazo.

\section{Infraestructura gris y movilidad}

Entre los objetivos de desarrollo sostenible se encuentra el de "lograr que las ciudades y los asentamientos humanos sean inclusivos, seguros, resilientes y sostenibles". Las ciudades se han convertido en el hábitat predilecto de la sociabilidad humana pero también son las responsables del consumo del $80 \%$ de la energía y de la emisión del 70\% de los gases de efecto invernadero (ONU, 2015). Uno de los elementos que contribuye a agravar la polución urbana es precisamente la movilidad. Los desarrollos urbanos descentralizados y de baja densidad han promovido un modelo de movilidad dependiente del vehículo privado que es un elemento de exclusión social por su alto coste de adquisición y mantenimiento (Herce, 2009).

La movilidad sostenible tiene como objetivo principal garantizar un transporte seguro, eficiente e inclusivo, basado en la complementariedad multimodal entre sistemas y la integración en el patrón funcional del suelo. Algunas ciudades han apostado por la mejora de la red de transporte masivo y por la planificación integral de la movilidad y los usos del suelo. En definitiva, la promoción de un modelo urbano compacto y mixto, en el que el transporte masivo tenga un papel vertebrador, no sólo de la movilidad, sino también de la forma de la ciudad. La intensificación de los usos del suelo en el entorno de las estaciones ferroviarias es una de las estrategias más comunes (Calthorpe, 1993).

\section{Caso de estudio. Lima}

La población actual de Lima Metropolitana es aproximadamente 9,5 millones de habitantes y se estima que en 2025 supere los 11 millones mientras que los viajes podrían incrementarse en un 50\% (INEI, 2018). Este incremento de la demanda de movilidad repercutirá en la calidad del transporte y vida de los limeños. El uso del vehículo privado, además de ser un factor de exclusión social y alto impacto ambiental, 

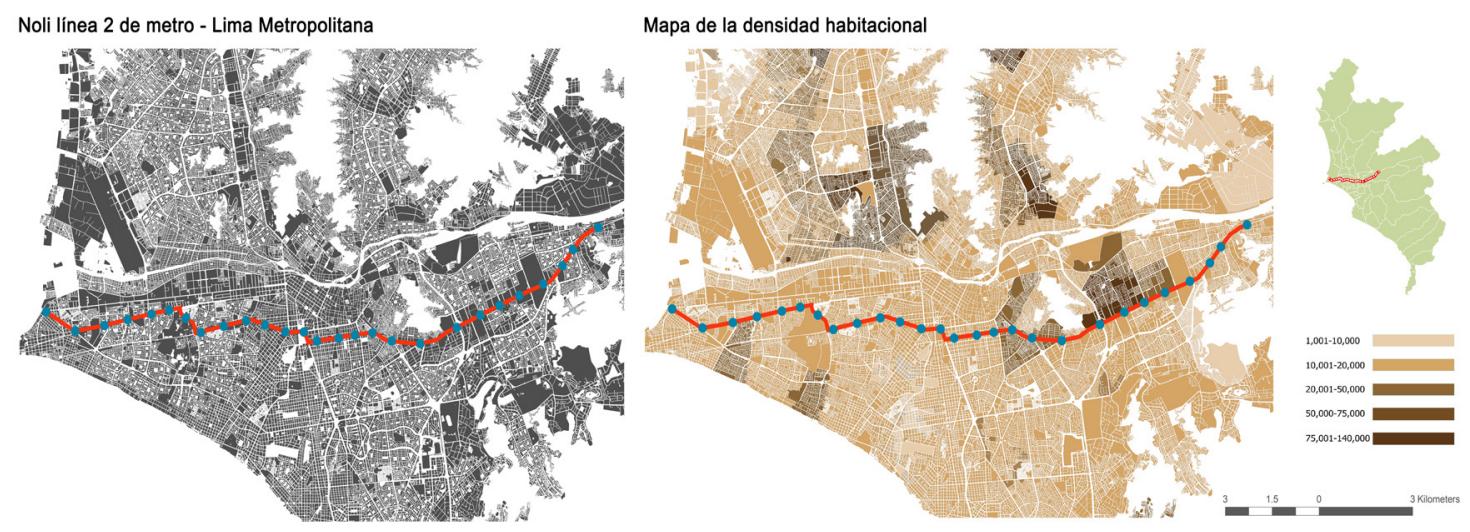

Fig. 1. Noli de la línea 2 de Metro y mapa de densidad habitacional en Lima Metropolitana. Fuente: Elaborado por Carlos Guardia Brown a partir de imágenes satelitales Google.

incrementa la congestión viaria y la ocupación en los centros urbanos, en detrimento de sistemas masivos, cuya cuota alcanza el $75 \%$ de los viajes de la conurbación, a pesar de ser considerado por los ciudadanos de Lima como el segundo mayor problema que afecta a su calidad de vida (IOP-PUCP, 2018).

En 2016, la contaminación en zonas urbanas y rurales provocó la muerte de 4,2 millones de muertes prematuras en todo el mundo. Lima fue la segunda ciudad con mayor índice de contaminación de América Latina, con $88 \mathrm{u} / \mathrm{m} 3$ de PM10 (OMS, 2016) y es considerada como el segundo problema ambiental más grave para el 72\% de la población (IOP-PUCP, 2018).

Uno de los factores que contribuye a mitigar los efectos de la contaminación atmosférica es la existencia de un sistema de espacios libres conectados y biodiversos que ponga en relación los espacios naturales y vacíos urbanos. En el caso de Lima, la superficie de áreas verdes por habitante es de $2,89 \mathrm{~m} 2$, dato que queda muy lejos de la recomendación de la OMS de 9-10 m2/habitante. De acuerdo con esta recomendación, la superficie de parques y jardines de Lima debería ser de 79 millones de metros cuadrados, de los cuales en la actualidad hay tan sólo 24,7 en sus 43 municipios (Lima como vamos, 2018).

\section{Antecedentes históricos}

En relación a la red de transporte público, el primer sistema de transporte masivo data de 1875 con la inauguración del Ferrocarril eléctrico Lima-Callao, pero no será hasta 1921 cuando se inaugure la primera línea de bus urbano (ómnibus). En los años 60, tanto la red de tranvías como las empresas de gestión del servicio formal de autobuses quiebran como consecuencia de la proliferación de la oferta de transporte informal que en los años 80 alcanza el $90 \%$. Uno de los males endémicos del sistema de transporte de Lima es un sector de transporte liberalizado con un sistema de tarifas desregularizada y $\sin$ restricciones espaciales al paso de todo de tipo de vehículos. En relación a los espacios abiertos, aunque el concepto de espacio verde a nivel legal se introduce con el Plan Piloto de 1949 (PPL), serán los Estudios de la Oficina Nacional de Planeamiento y Urbanismo de 1954 y 1958 los que constituyan el primer esfuerzo para el establecimiento de áreas verdes jerarquizadas como parte de una estructuración sistémica. Por vez primera y única, se establece un índice normativo de $8 \mathrm{~m} 2$ de área verde por habitante, y un sistema de grandes parques y vías parques que enlazan las principales zonas recreativas de la ciudad. El Plan de Desarrollo Metropolitano de Lima Callao, PLANDEMET (1968-1980), genera un primer diagnóstico recreacional en base al tamaño de las áreas verdes, la factibilidad de afectación y/o implementación, y funciones a cumplir de los sectores identificados, los cuales abarcan una distancia aproximada de recorrido a pie de $2.5 \mathrm{~km}$ y estarán definidos por la trama viaria. Se define así por vez primera, un sistema articulado de áreas verdes con diversas funciones y categorías pensadas para 
satisfacer las necesidades de recreación activa y pasiva de toda la población metropolitana. Sin embargo, la situación caótica en la que estaba sumida Lima en los 80, con un agotamiento de sus posibilidades funcionales y económicas en su estructura espacial, hizo que las áreas verdes dejaran de tener un espacio prioritario y fueran vistas de un modo más individualista (Guerra, 2012).

\section{Actualidad}

La creación del Instituto Metropolitano Protransporte en 2002 supuso un cambio de paradigma en el desarrollo y gestión de la red de transporte masivo. La aprobación del Plan Maestro Lima-Callao (JICA) en 2005, impulsó la construcción de la primera línea de BRT conocida como el Metropolitano y la ampliación de la Línea 1 de Metro de Lima con $34 \mathrm{~km}$ y 26 estaciones. Esta red básica se completó en diciembre de 2010 con la aprobación del Decreto Supremo n 059-2010MTC que configuraba una red de transporte metropolitana integrada entre Lima y Callao. En la actualidad, a pesar de ser una ciudad con una tasa de motorización muy baja $(1,4$ automóviles por cada diez habitantes), la emisión de gases de efecto invernadero por parte del transporte terrestre es el $39,8 \%$ del total (IOP-PUCP, 2018).

En el año 2014-15, la Municipalidad Metropolitana de Lima desarrolló el Plan de Espacios Abiertos e Infraestructura Ecológica (PEAIE) de Lima. La propuesta entendía el sistema metropolitano de espacios abiertos como una "infraestructura verde", que aportaba servicios ecológicos para la ciudad además de fortalecer el rol de los espacios públicos como dinamizadores de la vida social de la ciudad. Sin embargo, la debilidad institucional en materia de planificación urbana hacía y hace necesaria la aproximación estratégica al territorio, considerando los proyectos y propuestas en tanto que catalizadores capaces de introducir en el tejido urbano dinámicas que contribuyan a mejorar la calidad de vida de la ciudad (Olivares, 2017).

\section{La línea 2 de Metro de Lima como sistema ecológico}

La línea 2 de Metro de Lima como sistema eEl transporte público es uno de los factores principales en la pérdida de calidad de vida de los 9,5 millones de habitantes que configuran Lima Metropolitana. Con el objetivo de mejorar su movilidad, las administraciones públicas han promovido la construcción de infraestructuras como viaductos, puentes, enlaces viarios o intersecciones a desnivel, lo que ha alterado la realidad física, ambiental y social de los espacios por los que discurren. Este mismo efecto es el que se ha generado con la implantación de la línea 1 de Metro: disfunciones físicas en la estructura urbana por el efecto barrera de su infraestructura, pero también sociales, por la segregación que dicha infraestructura genera en los barrios. La visión sectorial de la red de transporte como

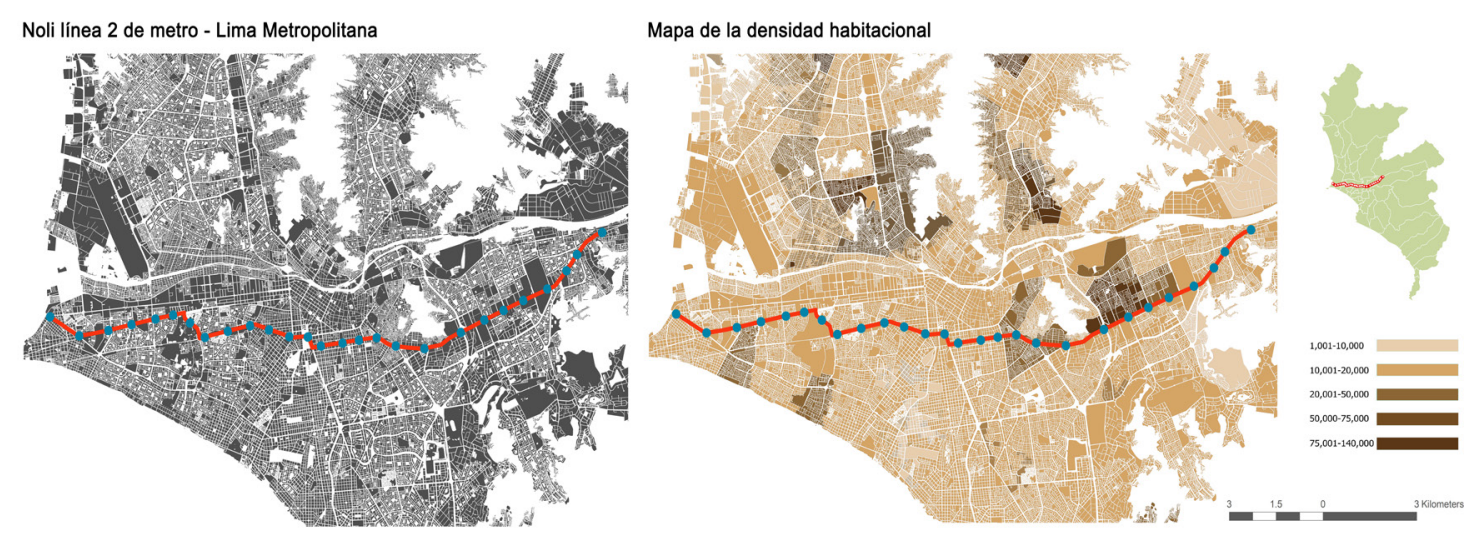

Fig. 2. Mapas de Ingreso per cápita y área verde por habitante y de la red de metro de Lima. Fuente: Elaborado por Carlos Guardia Brown a partir de imágenes satelitales Google. 
sistema autónomo, cuyo diseño depende de las solicitaciones técnicas de circulación con independencia del medio físico en el que se implanta, es la causa de este hecho.

Por otro lado, la línea 2 de Metro de Lima unirá de forma subterránea el distrito de Ate con Cercado del Callao, y se conectará con la línea 1 de Metro en la Estación central de la Plaza Grau. Se estima un potencial de 600.000 pasajeros diarios y un ámbito de influencia del $35 \%$ de la población de Lima Metropolitana. Asimismo, su implantación implicará una reducción del $10 \%$ de la circulación de vehículos privados (34.975 unidades), un factor relevante para una ciudad que niveles de polución atmosférica superiores a las recomendaciones de la OMS (IOP-PUCP, 2018).

Entre los objetivos del desarrollo urbano para la ciudad de Lima está la mejora de la accesibilidad a los servicios públicos básicos, el uso del territorio en armonía con los ecosistemas y la implementación de un sistema de movilidad integrada, sostenible e inclusiva. En la consecución de estos objetivos juega un papel clave el diseño de un espacio público de calidad para la interacción social en el que los desplazamientos no motorizados son prioritarios. En este sentido, la línea 2 de Metro puede ser una oportunidad para la creación de espacios abiertos de calidad en el entorno de sus estaciones, constituyendo un nuevo sistema conectado e inclusivo a escala metropolitana.

\section{Conclusiones}

La movilidad urbana es uno de los factores clave en la mejora de la calidad de vida y prosperidad económica de las áreas urbanas latinoamericanas. Los modelos de ordenación de baja densidad han fomentado el uso del vehículo privado y, en consecuencia, de sus externalidades negativas: contaminación atmosférica y acústica, congestión, sedentarismo, etc. Con el objetivo de mitigar estos efectos sobre los ciudadanos, las políticas públicas en las grandes conurbaciones latinoamericanas se han orientado a la promoción de sistemas de transporte masivo integrados en un sistema de espacios públicos. Lima Metropolitana ha apostado por la reducción de la congestión viaria en un horizonte del $10 \%$ a partir de la construcción de sistemas masivos de transporte como el Metropolitano (BRT) o la red de Metro. La construcción de la Línea 2 de Metro de Lima podría representar una oportunidad para la creación de un sistema de espacios conectados, accesibles y de calidad.

\section{Agradecimientos}

La documentación gráfica de la presente comunicación ha sido elaborada por Carlos Guardia Brown estudiante de pregrado en Geografía de la Pontificia Universidad Católica del Perú (PUCP) de Lima a quien agradecemos su compromiso con el proyecto.

\section{Referencias}

Barbosa et al. 2007. "Who benfits from access to green space? A case study from Sheffield, UK". Landscape and Urban Planning (83): 187-195. doi:10.1016/j. landurbplan.2007.04.004.

Bertolini, Luca.; et al. 2009. Transit oriented development: making it happen. Farnham (Reino Unido): Ashgate.

Calthorpe, Peter, et al. 1993. The next American Metropolis: ecology, community, and the American dream. Poticha, Shelley (coord.). New York: Princeton architectural press.

Cruz, J.; De Olivera, G.; Ramos, J.S. 2017. "El espacio libre en la planificación territorial: análisis comparado de las áreas metropolitanas en España". Ciudad y Territorio, Estudios Territoriales, (193): 401-416.

EEA, European Environment Agency (2011). Green infrastructure and territorial cohesion - The concept of green infrastructure and its integration into policies using monitoring systems. https://www.eea.europa.eu/ publications/green-infrastructure-andterritorial-cohesion (consultada el 26 de junio de 2019)

Feria, J.M. y Santiago, J. 2009. "Funciones ecológicas del espacio libre y planificación territorial en ámbitos metropolitanos. Perspectivas teóricas y experiencias recientes en el contexto español". Revista Electrónica de Geografía y Ciencias Sociales (vol XIII, 
299). doi: $10.1344 / \mathrm{sn} 2009.13 .1605$.

Figueroa,I. 2009. "Conectividad y accesibilidad de los espacios abiertos urbanos en Santiago de Chile." Tesis de Magíster, Instituto de Estudios Urbanos y Territoriales, Pontificia Universidad Católica de Chile.

Forman, R.T.T. y Gordon, M. 1986. Landscape Ecology. New York: John Willey \& Sons.

Guerra, T. M. 2012. "Estructura y tendencias de las áreas verdes en normas y planes del período 1949-1990, en el contexto de la renovación urbana. El caso de Lima". Tesis de Maestría Universidad Nacional de Ingeniería.

Herce, Manuel. 2009. Sobre la movilidad en la ciudad: propuestas para recuperar un derecho ciudadano. Barcelona: Reverté, Estudios universitarios de arquitectura, $\mathrm{n}^{\circ}$ 18.

INEI, Instituto Nacional de Estadística e Informática. 2010. "Perú: Estimación y proyecciones de población departamental, por años calendario y edades simples 19952025”. Boletín Especial No 22.

MEA, Millenium Ecosystem Assessment. 2005. Ecosystem and Human Well-being: Current State and Trends. Washington: Island Press.

Natural England 2009. Green Infrastructure Guidance. http://publications. naturalengland.org.uk/publication/35033 (consultada el 20 de mayo de 2019).

ONU, Organización de Naciones Unidas. 2015. Ver_"Objetivos de desarrollo sostenible." - https://www.un.org/ sustainabledevelopment/es/objetivos-dedesarrollo-sostenible/ (consultada el 15 de mayo de 2019).

ONU, Organización de Naciones Unidas. 2016. Ver_"The New Urban Agenda." Quito (Ecuador): United Nations Conference on Housing and Urban Development - Habitat III.

Pons, B. 2016. "La infraestructura verde como base de la resiliencia urbana. Estrategias para la regeneración de corredores fluviales urbanos del Banco Interamericano de Desarrollo." Tesis de Doctorado. Universidad Politécnica de Madrid.

Thomas, K. y Littlewood, S. 2010. "From
Green Belts to Green Infrastructure? The Evolution of a New Concept in the Emerging Soft Governance of Spatial Strategies." Planning Practice \& Research, (25:2): 20322. Doi: $10.1080 / 02697451003740213$

Tratalos, J. etal. 2007. "Urban form, biodiversity and ecosystem services." Landscape and Urban Planning, (83): 308 317 . Doi: 10.1016/j.landurbplan.2007.05.003 